\title{
Ternary Polymer Blends as Model Surfactant Systems
}

\author{
Newell R. Washburn, ${ }^{\dagger, *}$ Timothy P. Lodge,, and Frank S. Bates ${ }^{*,+}$ \\ Department of Chemical Engineering and Materials Science, and Department of Chemistry, \\ University of Minnesota, Minneapolis, Minnesota 55455
}

Received: December 1, 1999; In Final Form: April 24, 2000

\begin{abstract}
An investigation of the ternary phase prism for low molecular weight poly(ethylene oxide), squalane, and poly(ethylene oxide- $b$-ethylenepropylene) is presented. Comparisons are made between this ternary polymer system and classic water/oil/surfactant mixtures to establish further a universal phase diagram description for amphiphilic systems. A combination of visual isothermal measurements, small-angle X-ray scattering, smallangle neutron scattering, and dynamical mechanical spectrometry was used to characterize phases and determine phase boundaries. A rich phase diagram was revealed, including most of the equilibrium liquid crystalline phases associated with diblock copolymers, regions of two-phase and three-phase coexistence, and a bicontinuous microemulsion. Differences between this polymer phase diagram and those from water/oil/ surfactant systems highlight the strong effect of water in the latter.
\end{abstract}

\section{Introduction}

There is a strong analogy between homopolymer/homopolymer/diblock copolymer blends and water/oil/surfactant systems. Both ternary phase diagrams are composed of two components that have net repulsive interactions and a third component, an amphiphile, which is soluble in both. At high amphiphile concentrations and low temperatures, a single phase is stable, containing A-rich and B-rich domains separated by the amphiphile at the interface. These domains often form a periodic microstructure to maximize contact between similar components. At low amphiphile concentrations, the ternary phase diagram is characterized by macrophase separation, where two or three fluid phases are in equilibrium.

The structural and thermodynamic behavior of diblock copolymers have been studied extensively. ${ }^{1,2}$ Block copolymers contain sequences of chemically distinct repeat units that are covalently linked. Because of the enthalpic penalty when dissimilar monomers come in contact, block copolymers tend to microphase separate at low temperatures; domains form that contain almost exclusively one type of monomer. The symmetry of these periodic structures is determined by the relative volume fraction of the two blocks. Equilibrium morphologies include lamellae, a bicontinuous cubic phase known as the gyroid, hexagonally packed cylinders, and cubic arrangements of spheres. ${ }^{3,4}$

The overall structure and thermodynamics of $\mathrm{A}-\mathrm{B}$ diblock copolymers are controlled by three parameters: the overall degree of polymerization, $N_{\mathrm{AB}}=N_{\mathrm{A}, \mathrm{b}}+N_{\mathrm{B}, \mathrm{b}}$, where $N_{\mathrm{A}, \mathrm{b}}$ is the number of repeat units in the A block; the volume fraction of block $\mathrm{A}, f=N_{\mathrm{A}, \mathrm{b}} / N_{\mathrm{AB}}$; and the monomer-monomer interaction parameter, $\chi \sim 1 / T$. The segregation strength of the two blocks is quantified by the ratio of the enthalpy of mixing $\Delta H \propto \chi$ to the translational entropy $\Delta S \propto \mathrm{N}_{\mathrm{AB}}{ }^{-1}$. At low values of $\chi N_{\mathrm{AB}}$ or high temperatures, the entropic contribution to the free energy dominates and a correlated but unstructured polymer

\footnotetext{
* Author to whom correspondence should be addressed.

$\uparrow$ Current address: National Institute of Standards and Technology, Gaithersburg, MD 20899.

$\doteqdot$ Department of Chemical Engineering and Materials Science.

$\S$ Department of Chemistry.
}

melt results. At high values of incompatibility or low temperatures, the enthalpic penalty for $\mathrm{A}-\mathrm{B}$ contact drives the system to a periodic, microphase-separated state. The transition from ordered to disordered states is known as the order-disorder transition $\left(\right.$ ODT), which occurs when $\chi_{\mathrm{ODT}} \cong 10.495 / N_{\mathrm{AB}}$ for symmetric diblock copolymers ${ }^{4}$ within mean-field theory.

Upon cooling, binary mixtures of homopolymers often undergo transitions from a single homogeneous phase at high temperature to a macrophase-separated state with two phases in equilibrium at low temperature. Mean-field theory predicts that homopolymers of equal molar volumes, $N_{\mathrm{A}, \mathrm{h}}=N_{\mathrm{B}, h}=N_{\mathrm{h}}$, have a critical composition at a volume fraction $\phi_{\mathrm{A}}=0.5$ and a critical temperature given by $\chi_{\text {crit }}=2 / N_{\mathrm{h}}{ }^{5}$ This critical point is known as the upper critical solution temperature. Specific attractive interactions between monomers can also lead to the opposite behavior, resulting in phase separation upon heating; this lower critical solution temperature is exhibited by, e.g., poly(vinyl methyl ether)/poly(styrene) ${ }^{6}$ and poly(methyl methacrylate)/poly(ethylene oxide $)^{7}$ blends.

The effect of added homopolymer on the phase behavior of ordered diblock copolymers depends strongly on the size of the homopolymer relative to the diblock copolymer, $\alpha_{\mathrm{A}}=N_{\mathrm{A}, \mathrm{h}} /$ $N_{\mathrm{AB}} \cdot{ }^{8-11}$ Calculations performed by Matsen ${ }^{12}$ indicate that, for $\alpha_{\mathrm{A}}=1$, added homopolymer A tends to segregate to the middle of the A-rich domain. The presence of this layer of homopolymer tends to enhance order in the microstructure and increases the degree of segregation. However, low molecular weight homopolymers $\left(\alpha_{\mathrm{A}}<1 / 4\right)$ tend to distribute more uniformly in a given domain due to a higher translational entropy. This dilutes the copolymer concentration and effectively reduces the degree of segregation. The homopolymers used in this work fall into the latter category, although differences in how they solvate the respective blocks may still be expected.

At constant pressure, the ternary phase diagram may be represented as a triangular prism with the corners of the base locating the three pure components and the vertical axis representing temperature. In this study we have characterized the phase diagrams produced along five planes of the phase prism in the poly(ethylene oxide)/squalane/poly(ethylene oxide)poly(ethylenepropylene) (PEO/PEP*/PEO-PEP) system, as 


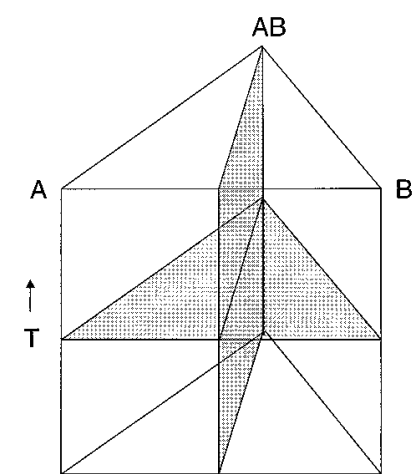

Figure 1. Isobaric phase prism for ternary systems. The shaded planes represent phase diagrams presented in this work. In addition, the three faces of the prism (the binary phase diagrams) were also characterized.

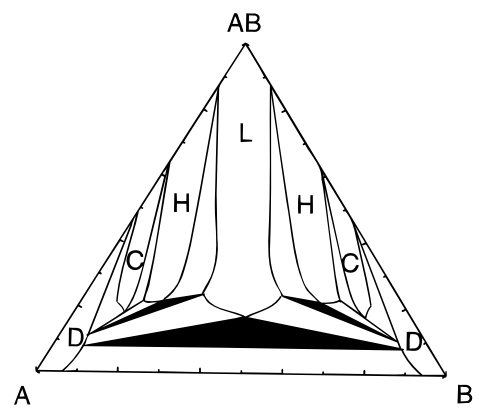

Figure 2. Isothermal homopolymer/homopolymer/diblock copolymer phase diagram calculated using mean field theory (after Janert and Schick ${ }^{17}$ ). Letters refer to the structure of the ordered phases: L (lamellar), $\mathrm{H}$ (hexagonally packed cylinders), C (cubic), and D (disordered), all separated by regions of phase coexistence. The darkened triangles represent three-phase equilibrium with the center triangle denoting coexistence between the lamellar phase and two homopolymer-rich phases.

shown in Figure 1. Throughout this report we refer to squalane, a 32-carbon saturated alkane as $\mathrm{PEP}^{*}$ since the molecular architecture of this oligomer is nearly identical to that of PEP. The phase diagrams determined include the vertical faces of the phase prism (three binary phase diagrams), the phase diagram obtained by varying the copolymer concentration and temperature at fixed $\mathrm{A} / \mathrm{B}$ ratio (referred to as the isopleth), and an isothermal phase diagram. These last two planes are shaded in Figure 1 for reference.

The isopleth for the homopolymer/homopolymer/diblock copolymer phase diagram has been characterized for several systems. ${ }^{14,15}$ At high concentrations of symmetric block copolymer $(f=1 / 2)$ the system is a disordered melt at high temperatures and adopts a lamellar structure at low temperatures. At low block copolymer concentrations, the system is also a disordered melt at high temperatures but macrophase separates into two homopolymer-rich phases at low temperatures. For $\alpha_{A}$ $=\alpha_{\mathrm{B}}=\alpha$, the transition from microphase to macrophase separation is predicted to occur at a total homopolymer volume fraction of $1 /\left(1+2 \alpha^{2}\right) .{ }^{16}$ This indicates that, as $N_{\mathrm{h}}$ increases relative to $N_{\mathrm{AB}}$, more diblock is required to solubilize the homopolymers into a single-phase microstructure.

The isothermal homopolymer/homopolymer/diblock copolymer phase diagram has been predicted theoretically ${ }^{17,18}$ but never fully characterized experimentally to the best of our knowledge. A ternary phase diagram calculated by Janert and Schick ${ }^{17}$ with $\alpha_{\mathrm{A}}=\alpha_{\mathrm{B}}=0.3$ and $\chi N_{\mathrm{AB}}=11.0$ is reproduced in Figure 2 . The symmetric diblock copolymer orders with a lamellar (L) structure. As equal volumes of the two homopolymers are added incrementally, the lamellar structure swells until the system phase separates into three coexisting phases, the lamellar phase and two homopolymer-rich solutions (D). The tip of the threephase window is predicted to lie at a composition containing less than $20 \%$ block copolymer. Below ca. $10 \%$ block copolymer the lamellar phase disappears and two-phase equilibrium is predicted, with the block copolymer segregating equally between the two phases.

As homopolymer $A$ is added to the diblock copolymer, the $A$-rich domains swell until a transition to a hexagonally ordered structure $(\mathrm{H})$ occurs at around $13 \%$ added homopolymer (the authors did not consider the gyroid phase in their work). As more homopolymer is added, a cubic phase (C) appears at $35 \%$ homopolymer and this disorders at 50\% homopolymer. Regions of two-phase coexistence separate regions characterized by a single microstructure; these are shown as unlabeled areas in Figure 2. Increasing $\chi N_{\mathrm{AB}}$ increases the composition range over which ordered microstructures are stable.

The main differences between the homopolymer/homopolymer/diblock copolymer blends and the water/oil/nonionic surfactant system can be attributed to the thermodynamic properties of water. Water and oil segregate strongly and fail to mix even at high temperature. By contrast, the critical point for phase separation in most polymer-polymer mixtures can be placed at an experimentally tractable temperature by adjusting the molecular weights; the monomers themselves are usually miscible. Furthermore, there is asymmetry in the way the surfactant interacts with water and oil, with a strongly temperature-dependent water-surfactant interaction. Addition of water to a disordered nonionic surfactant can drive the system to a liquid crystalline state to promote the segregation of the polar and nonpolar domains, ${ }^{19}$ whereas addition of oil does not. In mixtures of water and nonionic surfactant where the polar surfactant group is based on ethylene oxide, the two components are completely miscible at room temperature but phase separate at higher temperature. ${ }^{20}$ This "inverse" temperature dependence is also manifest in ternary mixtures with oil. At room temperature the nonionic surfactant preferentially segregates into the aqueous phase, whereas at higher temperatures the nonionic surfactant segregates into the oil phase. Over a narrow range of intermediate temperatures, the water and oil phases compete nearly equally for the amphiphile and a third phase is stabilized. This surfactant-rich phase is a microemulsion; near the middle of the temperature range over which the third phase is stable, a bicontinuous structure evolves to accommodate equal volumes of the two components. ${ }^{21}$ This bicontinuous microemulsion is a correlated, isotropic fluid with a spongelike structure. Recently, the bicontinuous microemulsion has been observed in polymer blends ${ }^{15,22}$ where it has been shown to be stable over a substantially wider temperature range.

In general, therefore, it is expected that polymeric systems can be less strongly segregated and that the temperature dependence of the interactions will be much weaker and more symmetric than for aqueous systems. In studying a ternary system in which the interactions between the amphiphile and other components are nearly equal and do not display strong temperature dependences, we seek to identify those elements of the water/oil/surfactant phase diagram that are due to the particular behavior of water, and those that are general features of ternary phase diagrams involving amphiphiles.

\section{Experimental Section}

Materials. Dimethyl poly(ethylene glycol) dimethyl ether $(\mathrm{PEO})\left(M_{\mathrm{n}}=500 \mathrm{~g} / \mathrm{mol}, M_{\mathrm{w}} / M_{\mathrm{n}}=1.1, \rho_{\mathrm{PEO}}=1.07 \mathrm{~g} / \mathrm{mL}\right)$ 
was purchased from the Aldrich Chemical Co. The viscous liquid had a noticeable yellow color, which was removed by dissolution in methylene chloride and passing over basic alumina. Most of the solvent was removed using a rotavap, and subsequently the polymer was dried overnight at $70{ }^{\circ} \mathrm{C}$ under vacuum while stirring rapidly. Squalane (PEP*, Aldrich, $\rho_{\text {PEP* }}$ $=0.81 \mathrm{~g} / \mathrm{mL}$ ), a saturated $\mathrm{PEP}^{*}$ hydrocarbon with six pendant methyl groups along the backbone, was purified by stirring vigorously over concentrated sulfuric acid. After $20 \mathrm{~min}$, the aqueous layer developed an intense orange color and the squalane was isolated using a separatory funnel. Residual acid was removed by triple washing with saturated sodium bicarbonate solutions. Residual sodium bicarbonate was then removed by triple washing with HPLC grade water (Omni). The purified squalane was dried by heating overnight at $70^{\circ} \mathrm{C}$ under vacuum while stirring rapidly. The purity of both "homopolymers" was verified using NMR and size exclusion chromatography (SEC). The poly(ethylene oxide- $b$-ethylenepropylene) (PEO-PEP) diblock copolymer used in this work was synthesized according to the procedure of Hillmyer and Bates. ${ }^{23}$ The PEP block has the same microstructure as squalane. Assuming the densities of the blocks are equal to those of the homopolymers, a volume fraction of $f_{\mathrm{PEO}}=0.50$ is calculated. From ${ }^{1} \mathrm{H}$ NMR the average composition of the diblock is $(\mathrm{EO})_{42}(\mathrm{EP})_{20}$ leading to a molecular weight of $M_{\mathrm{n}}=3000$; the polydispersity index $M_{\mathrm{w}} /$ $M_{\mathrm{n}}=1.2$ as measured by SEC. All materials were stored in amber bottles under a blanket of argon to prevent absorption of water. The compositions of polymer blends are reported in terms of mass fractions.

Oil Bath Measurements. Ampules were washed with concentrated nitric acid, triple rinsed with HPLC-grade water and dried at $150{ }^{\circ} \mathrm{C}$ for $1 \mathrm{~h}$. A total blend volume of $1.0 \mathrm{~mL}$ was loaded and the sample was frozen in liquid nitrogen and flame-sealed under vacuum. Samples were immersed in a Schott-Gerate oil bath, with a temperature stable to within \pm 0.1 ${ }^{\circ} \mathrm{C}$. Equilibration times for the homopolymer blends were relatively short, usually on the order of minutes. For the PEO/ $\mathrm{PEP}^{*}$ binary phase diagram, samples were equilibrated in the oil bath at low temperature. Below $100{ }^{\circ} \mathrm{C}$ all samples displayed two-phase coexistence and the relative volume fractions of the two phases was noted. The temperature was increased incrementally, allowing for equilibration at each temperature, and the samples were shaken vigorously. At highly asymmetric compositions, the relative volume of one phase was observed to decrease gradually as the temperature increased, in agreement with the lever rule for two-phase coexistence. The binodal was marked as the temperature at which the minority phase disappeared completely. For samples within $4 \%$ of the critical composition, samples became opalescent as the temperature was increased to within a few degrees of the spinodal and it became difficult to determine exactly when the two phases merged. This temperature was therefore taken where the meniscus disappeared. Similar difficulties were encountered when the line of critical points was mapped out as a function of added PEOPEP. However, even in these ternary blends equilibration times at temperatures greater than $170{ }^{\circ} \mathrm{C}$ were short.

In investigating phase equilibrium in ternary blends at lower temperatures, longer equilibration times were necessary, ranging from at least 3 days at $100{ }^{\circ} \mathrm{C}$ to 7 days at $60{ }^{\circ} \mathrm{C}$ for samples with less than $3 \%$ diblock. Blends with higher concentrations of diblock were often allowed to anneal for at least 2 weeks. When the samples were purified following the methods described in the Materials section, no evidence for degradation was observed by visual observations or GPC.
Dynamical Mechanical Spectrometry. Rheological measurements were performed using a Rheometrics Dynamic Stress rheometer. The parallel plate geometry was employed using 25 $\mathrm{mm}$ diameter, electrically heated plates with a constant flow of nitrogen to prevent sample degradation. Samples were loaded at $200{ }^{\circ} \mathrm{C}$ and cooled to $100{ }^{\circ} \mathrm{C}$ at which point the gap between the plates was lowered to give a nearly vertical sample edge. The sample was then equilibrated for $2 \mathrm{~h}$. Temperature ramps from 100 to $170{ }^{\circ} \mathrm{C}$ were performed at a heating rate of $1{ }^{\circ} \mathrm{C} /$ min, a strain amplitude of less than $2 \%$, and a frequency of 1 $\mathrm{rad} / \mathrm{s}$.

Small-Angle X-ray Scattering. Small-angle X-ray scattering (SAXS) measurements were made using a small-angle beamline at the University of Minnesota; this instrument is described elsewhere. ${ }^{24}$ Samples were prepared by blending the appropriate polymers in scintillation vials using a few drops of methylene chloride to facilitate complete mixing. The viscous liquid was transferred by pipet to nitric acid-washed $2 \mathrm{~mm}$ quartz capillaries and the methylene chloride was removed by placing the samples in a vacuum oven for 4 days at $80{ }^{\circ} \mathrm{C}$. The mass of selected samples was monitored over time and reached a constant value after 3 days, indicating the methylene chloride had been removed. The polymer blend creeps up the sides of the capillary during this time and a centrifuge was used to drive the sample to the bottom. Order-disorder transition temperatures $\left(T_{\mathrm{ODT}}\right)$ were measured by increasing the heating block temperature incrementally, until the disappearance of higher order peaks and a concomitant broadening of the first-order peak was observed.

Small-Angle Neutron Scattering. SANS experiments were performed at the National Institute for Standards and Technology in Gaithersburg, MD, on the NIST/Exxon/University of Minnesota $30 \mathrm{~m}$ instrument using $\lambda=7 \AA$ wavelength neutrons $(\Delta \lambda / \lambda=0.11)$. These experiments relied on the small, but sufficient, scattering contrast between PEP (or PEP*) and PEO. Samples were loaded in quartz cells with a path length of 1.05 $\mathrm{mm}$. All of the samples produced nearly azimuthally isotropic two-dimensional scattering patterns that were averaged to onedimensional plots of intensity $(I)$ versus $q$, the magnitude of the scattering wave vector $(q=4 \pi \sin (\theta / 2) / \lambda$ where $\theta$ is the scattering angle). The scattering data were corrected for background and cell scattering, detector sensitivity, sample thickness, and transmission, and then converted to an absolute differential scattering cross section per unit sample volume $\left(\mathrm{cm}^{-1}\right)$ using previously reported calibration techniques. ${ }^{25}$

\section{Results and Analysis}

General. In the phase diagrams with block copolymer as one component, regimes of coexistence between ordered microstructures are not depicted in the figures because only isolated examples of coexistence were observed experimentally. Phase diagrams involving ordered microstructures were investigated using SAXS and SANS and, in some samples, a second firstorder peak was observed, either as a distinct peak or as a shoulder. Indexing was performed by assigning higher order peaks to the appropriate first order peak. It was generally possible to assign all peaks to one of two phases. On the basis of these experiments is it concluded that the coexistence regions typically span less than $4 \%$ in composition, but for the density of points taken it was not possible to demarcate the regions of coexistence clearly.

Binary Phase Diagrams. The PEO/PEP* phase diagram shown in Figure 3 was established using oil bath measurements described in section 2 . The coexistence curve is nearly symmetric, suggesting that the reversible work required to move 


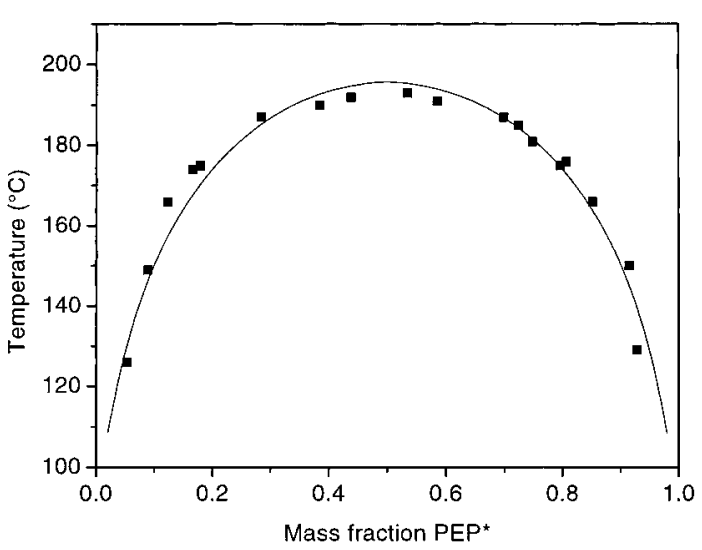

Figure 3. The PEO/PEP* coexistence curve as determined by visual isothermal measurements. Data points represent actual samples measured while the solid line is the fit to Flory-Huggins theory converted to PEP* mass fraction.

one PEO molecule from the PEO-rich phase to the PEP*-rich phase is nearly the same as that required to move one PEP* molecule from the PEP*-rich phase to the PEO-rich phase. The upper solution critical temperature (UCST) is $192 \pm 2{ }^{\circ} \mathrm{C}$ at a PEP* mass fraction of $0.49 \pm 0.03$. This corresponds to a critical PEP* volume fraction of $\phi_{\text {PEP* }}=0.42 \pm 0.03$, assuming $\rho_{\text {PEP* }}$ $=0.81$ and $\rho_{\text {PEO }}=1.07 \mathrm{~g} / \mathrm{mL}$. The binodal is well-fit using Flory-Huggins theory ${ }^{26}$ with a $\chi$ parameter of

$$
\chi=472 / T-0.76
$$

where $T$ is the temperature in kelvin and the second term represents the standard "entropic" contribution.

Based on SAXS, the pure diblock has a lamellar microstructure. The lamellar lattice spacing, $d^{*}=2 \pi / q^{*}$ where $q^{*}$ is the scattering wave vector associated with the principal reflection, is $94 \AA$ at $80{ }^{\circ} \mathrm{C}$. An ODT of $164{ }^{\circ} \mathrm{C}$ was determined using dynamical mechanical spectrometry and SAXS. Figure $4 \mathrm{a}$ illustrates the nearly discontinuous drop in the dynamic elastic shear modulus as a specimen is heated through the first-order ODT. SAXS confirms the ODT, as shown in Figure 4b. Below $160{ }^{\circ} \mathrm{C}$, the principal reflection is clearly observed in the scattering patterns but the peak height decreases and peak width increases abruptly at $175^{\circ} \mathrm{C}$, and by $180{ }^{\circ} \mathrm{C}$ very little scattering is observed on the scale of this figure. The reason for the small difference between the ODT's measured using SAXS and dynamical mechanical spectrometry is not clear.

The PEP*/PEO-PEP phase diagram was also investigated using SAXS. PEP* should selectively segregate into the PEP block at lower temperatures. Added PEP* swells the PEP block, thereby increasing the curvature at the PEO-PEP interface and inducing changes in microstructure. Most of the equilibrium microstructures associated with diblock copolymer melts were observed as a function of increasing PEP* concentration, including L (Figure 5a), G (gyroid, Figure 5b), and H (Figure $5 c)$. The only evidence for cubic arrays of spheres (C) was found in samples that contained coexisting cylinders and spheres (Figure 5d). The principal peak for the spheres appears as a shoulder on the low $q$ side of the principal $\mathrm{H}$ peak, consistent with a decrease in the concentration of diblock in the cubic phase leading to an increase in principle lattice spacing. Note that the clear evidence of the $\sqrt{ } 7$ peak of spheres confirms that it is body-centered cubic structure, not face-centered or simple cubic. The resulting PEP*/PEO-PEP phase diagram is shown in Figure 6a. The lamellar microstructure of the pure diblock is retained above $18 \%$ PEP*, but by $27 \%$ PEP*, the PEP* block
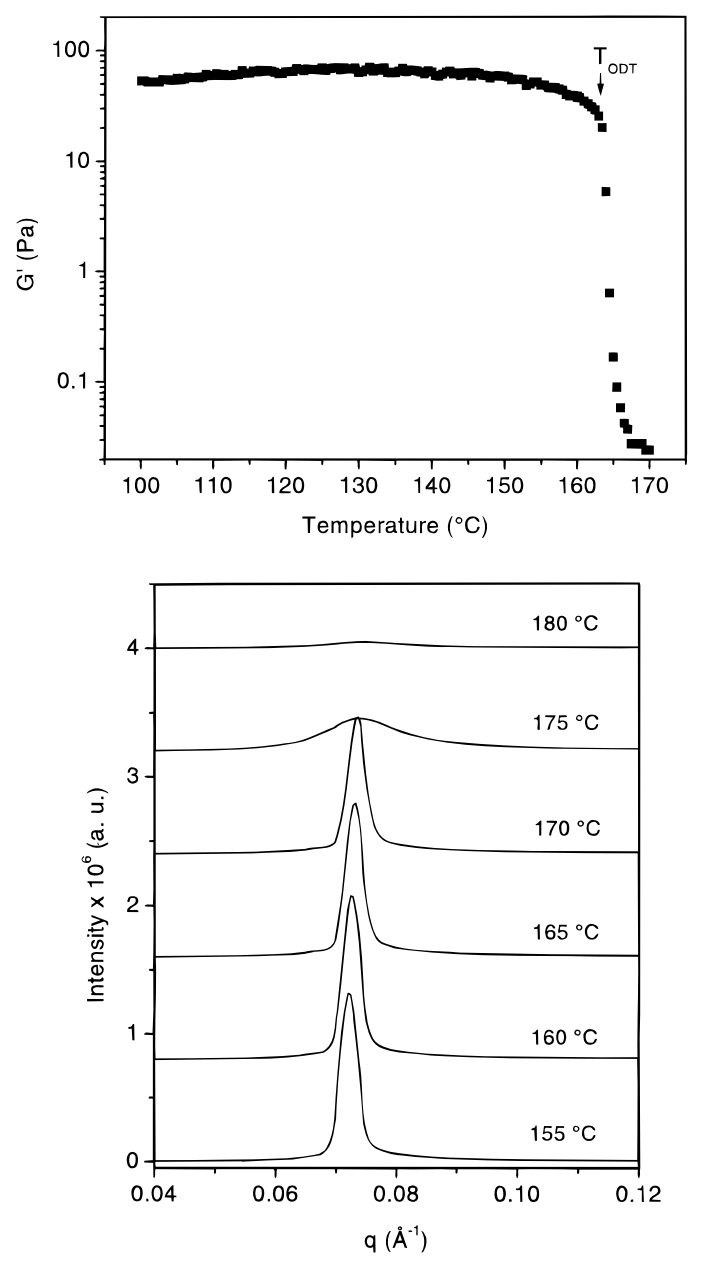

Figure 4. (a) Dynamic shear elastic modulus for the PEO-PEP diblock as a function of temperature. (b) SAXS patterns taken in $5{ }^{\circ} \mathrm{C}$ increments for the PEO-PEP diblock. Curves are offset for clarity.

has swelled enough to induce a nonzero mean curvature and the system adopts the gyroid structure. By $37 \%$ PEP*, the increase in curvature leads to the formation of a hexagonally packed cylinder phase, which persists to at least 55\% PEP*. The $T_{\text {ODT }}$ 's, measured using SAXS experiments as described in section 2, are relatively constant as more $\mathrm{PEP}^{*}$ is added to the blend. At 58\% PEP*, coexistence between hexagonal and cubic phases was observed at lower temperatures. The peaks assigned to $\mathrm{C}$ disappeared at $108^{\circ} \mathrm{C}$ upon heating whereas the peaks associated with $\mathrm{H}$ persisted until $122^{\circ} \mathrm{C}$. We were unable to prepare a sample that displayed only the scattering associated with bcc spheres, and so we conclude that the $T_{\mathrm{ODT}}$ 's decrease sharply as more PEP* is added. At lower concentrations of block copolymer, only micellar solutions were observed. However, crystallization of the diblock PEO restricts the temperatures investigated to above $70{ }^{\circ} \mathrm{C}$. No order-order transitions were recorded as a function of temperature, suggesting that the coexistence regions between ordered phases are nearly vertical in the composition-temperature phase diagram.

The PEO/PEO-PEP phase diagram, determined using the same methods as for the PEP*/PEO-PEP phase diagram, is shown in Figure 6b. The phase diagram is similar to that for PEP*/PEO-PEP, with slight differences in the compositions at which the structure evolves from lamellar to gyroid to hexagonally packed cylinders. The $T_{\mathrm{ODT}}$ 's appear to decrease more rapidly with homopolymer addition than when the diblock is blended with PEP*, suggesting that PEO is a slightly better solvent for the diblock than PEP*. Overall, however, these 

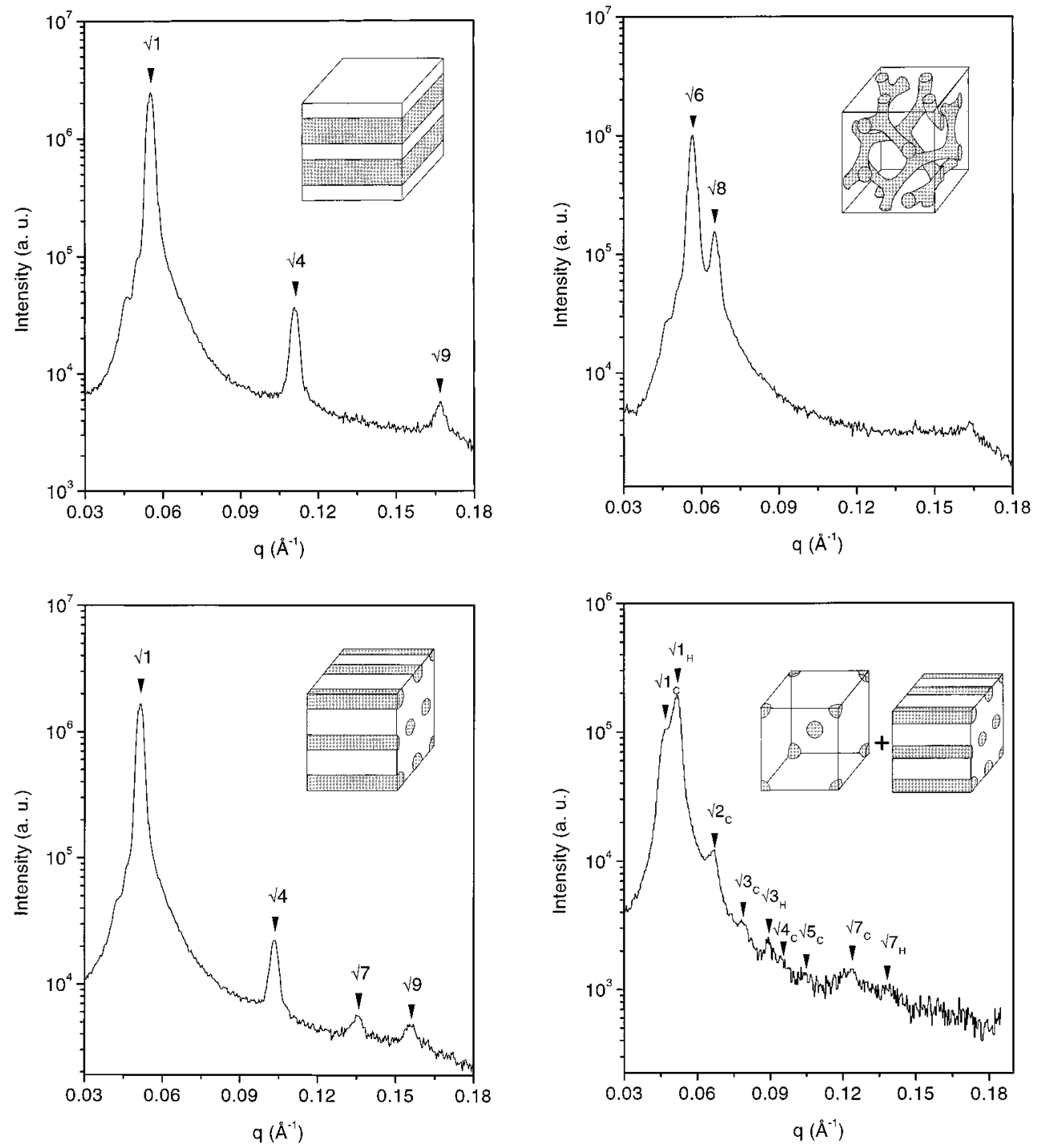

Figure 5. SAXS patterns for the PEP*/PEO-PEP blends representative of (a) lamellar (9\% PEP*), (b) gyroid (27\% PEP*), (c) hexagonally packed cylinders (36\% PEP*), (d) coexistence between cylinders and bcc spheres (55\% PEP*). The numbers over each peak are the square root of the sum of the squares of the Miller indices. The subscripts in (d) refer to the phase to which the peak was assigned with $\mathrm{C}$ denoting cubic and $\mathrm{H}$ denoted hexagonally ordered cylinders.

phase diagrams are quite similar, indicating that the homopolymer/diblock copolymer interactions are approximately symmetric.

Isopleth Phase Diagram. A summary of the isopleth data for the PEO/PEP*/PEO-PEP system is shown in Figure 7. Below $8 \%$ block copolymer, multiphase equilibrium between isotropic fluid phases was observed using visual isothermal measurements. Between 9 and 11\% PEO-PEP, coexistence between a bicontinuous microemulsion and lamellae was identified via SANS at lower temperatures. Above $11 \%$ block copolymer, the only equilibrium microstructure observed was L and the $T_{\mathrm{ODT}}$ 's increase monotonically as the block copolymer concentration increases. This diagram is similar to diagrams shown in previous work, ${ }^{14,15,22,35}$ but it contains features such as three-phase coexistence and a region of phase coexistence between bicontinuous microemulsion and lamellae. Further comparisons between this phase diagram and diagrams previously observed in homopolymer/homopolymer/diblock copolymer blends and water/oil/surfactant systems will be made in the discussion section.

Visual isothermal measurements were conducted to characterize the isopleth at diblock mass fractions below 9\%. Above 192
${ }^{\circ} \mathrm{C}$, all samples were clear, colorless, and single-phase. As the temperature was lowered, phase separation occurred, with the transition temperature along the isopleth decreasing from 192 ${ }^{\circ} \mathrm{C}$ for the pure homopolymer blend to $173{ }^{\circ} \mathrm{C}$ with $8 \%$ diblock added. All samples underwent transitions from a single phase to two clear, colorless phases of approximately equal volume and displayed critical opalescence near the demixing temperature, indicating that the value of the critical composition does not change significantly as diblock is added. The relative volume of the PEO-rich phase was found to increase as the temperature was lowered to $120^{\circ} \mathrm{C}$, suggesting that the diblock copolymer is nearly equally distributed between the two phases at high temperatures but segregates preferentially into the PEO-rich phase as the temperature decreases. With $8 \%$ block copolymer at $120{ }^{\circ} \mathrm{C}$, the lower phase occupies approximately $60 \%$ of the total volume. This is consistent with the conclusion drawn from the analysis of the two homopolymer/diblock copolymer phase diagrams (Figures 6a and (b)) that PEO is a slightly better solvent for PEO-PEP than PEP*. This is also reflected as asymmetry in the PEO/PEP* coexistence curve when it is plotted as a function of volume fractions, i.e., the critical volume fraction of PEO is about 0.42 . 

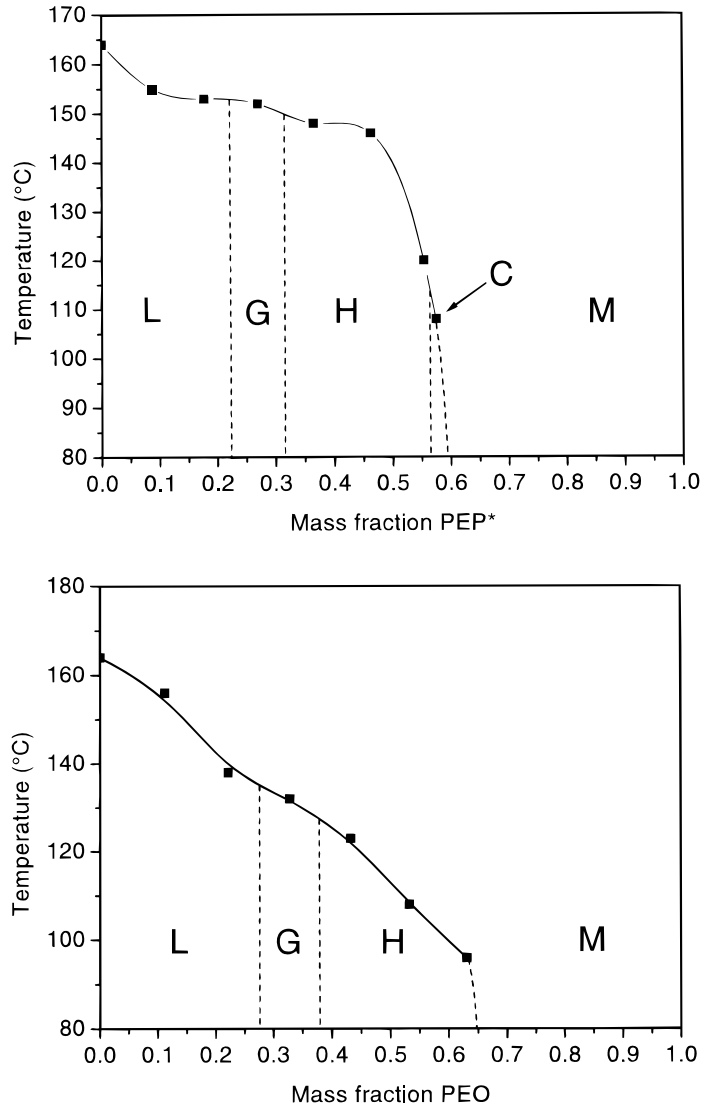

Figure 6. (a) The PEP*/PEO-PEP phase diagram. Dashed lines represent approximate phase boundaries. (b) The PEO/PEO-PEP phase diagram.

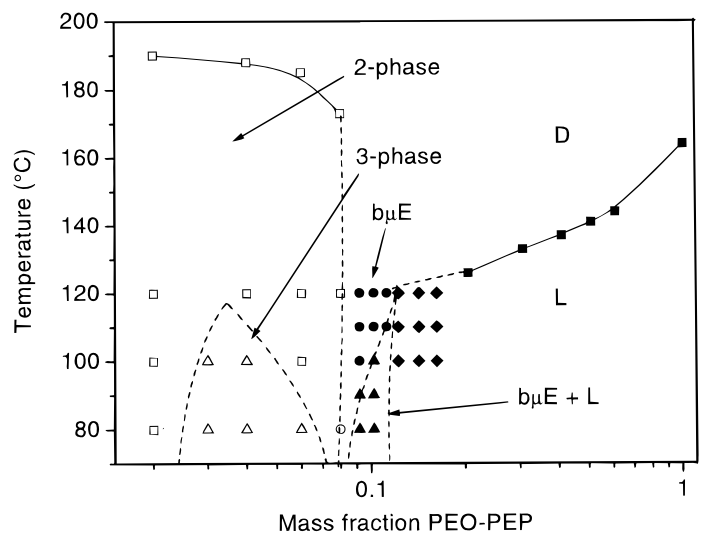

Figure 7. Isopleth for the PEO/PEP*/PEO-PEP system. Below $9 \%$ block copolymer and $192{ }^{\circ} \mathrm{C}$, the system exhibits multiphase equilibrium between isotropic fluids. The open circle at $80{ }^{\circ} \mathrm{C}$ represents the observation of a single phase (bicontinuous microemulsion), open squares denote the observation of 2-phase equilibrium, and open triangles denote three-phase equilibrium. The bicontinuous microemulsion forms a channel in the phase diagram that narrows as the temperature is lowered from 120 to $80^{\circ} \mathrm{C}$. Filled circles represent the observation of scattering attributable to the bicontinuous microemulsion only while filled triangles represent bicontinuous microemulsion and lamellar scattering. Filled diamonds represent SANS data attributed to lamellae and filled squares represent lamellae ODTs as determined using SAXS.

At $100{ }^{\circ} \mathrm{C}$, three distinct phases were observed in certain $\mathrm{PEO} / \mathrm{PEP} * / \mathrm{PEO}-\mathrm{PEP}$ mixtures. The three-phase samples have two clear phases and a slightly turbid phase in between. At 80 ${ }^{\circ} \mathrm{C}$ the three-phase region spanned a broader composition range, suggesting PEO becomes a worse solvent for PEO-PEP as the temperature is reduced. This continued growth of the three- phase region also indicates the three-phase window spans a wide range in temperature. Three-phase equilibrium commonly has been observed in surfactant systems but to our knowledge this is the first report of three-phase equilibrium in a polymer blend. While not surprising, there are some important differences between the temperature dependence of the polymer three-phase window and that in the water/oil/surfactant systems that will be considered in the discussion section.

To elucidate the phase behavior of samples along the isopleth with 9-20\% block copolymer, small-angle neutron scattering experiments were performed. The temperature dependence of the sample with 9\% PEO-PEP is shown in Figure 8a. At 110 ${ }^{\circ} \mathrm{C}$, a single asymmetric peak centered at $0.0094 \AA^{-1}$ is observed. The scattering is characteristic of a bicontinuous microemulsion. Teubner and Strey $^{27}$ developed an empirical model based on a Ginsburg-Landau approach ${ }^{28}$ to fit the scattered intensity $I(q)$ from such a mixture:

$$
I(q)=\frac{\kappa}{a_{2}+c_{1} q^{2}+c_{2} q^{4}}
$$

where $\kappa$ is a constant that accounts for the sample scattering contrast, and $a_{2}, c_{1}$, and $c_{2}$ are adjustable parameters. The fit in Figure $8 \mathrm{a}$ was only taken over the region marked by the solid line. As evidenced by the dashed line, the intensity at higher $q$ is slightly larger than predicted by the Teubner-Strey model. This has been observed previously in other polymeric bicontinuous microemulsions ${ }^{15}$ and is attributed to, inter alia, intrachain scattering when $q R_{\mathrm{g}}>1$, where $R_{\mathrm{g}}$ is the polymer radius of gyration. At $100{ }^{\circ} \mathrm{C}$ the peak becomes sharper and continues to sharpen as the temperature is decreased. However, at $90{ }^{\circ} \mathrm{C}$ a second peak appears at higher $q$, and by $80^{\circ} \mathrm{C}$, the second peak is clearly visible. Interestingly, at $80{ }^{\circ} \mathrm{C}$ the ratio of the second peak position to the first is close to $2: 1$, which might suggest the second peak is a higher order reflection. An amphiphilicity factor, $f_{\mathrm{a}}=c_{1} /\left(4 a_{2} c_{2}\right)^{1 / 2}$, may be defined which is a measure of the structure of the microemulsion. ${ }^{21}$ At 110 ${ }^{\circ} \mathrm{C}$ and $9 \%$ PEO-PEP, the amphiphilicity factor is calculated to be -0.28 , whereas at $80{ }^{\circ} \mathrm{C}, f_{\mathrm{a}}=-0.93$, both consistent with a "good" or highly structured microemulsion. ${ }^{3}$ For comparison, $f_{\mathrm{a}}=-1$ indicates the system has adopted a lamellar structure and $f_{\mathrm{a}}=+1$ indicates that the liquid is completely disordered.

Scattering patterns from the sample containing $10 \%$ block copolymer are shown in Figure $8 \mathrm{~b}$. A progression from a single peak at higher temperatures to two peaks at lower temperatures is again observed, except in this sample the high- $q$ peak is first apparent at $100{ }^{\circ} \mathrm{C}$ instead of $90{ }^{\circ} \mathrm{C}$. At $80{ }^{\circ} \mathrm{C}$ the positions of the first and second peak are the same in both the $9 \%$ and $10 \%$ samples although the intensity of the second peak is now greater than that of the first peak in the latter. The solid curve represents an optimized fit of eq 2 to the low- $q$ portion of the SANS results.

Scattering from the $11 \%$ sample shown in Figure $8 \mathrm{c}$ is quite different, with only one peak evident at each measurement temperature. However, on the basis of the progression of results beginning with the $9 \%$ mixture, we associate the scattering at $110{ }^{\circ} \mathrm{C}$ with a bicontinuous microemulsion and fit the data with eq 2 , whereas the peaks at 100,90 , and $80^{\circ} \mathrm{C}$ are classified with the higher $q$ peaks seen in Figure $8 \mathrm{a}$ and b. With $12 \%$ block copolymer (shown in Figure 8d), only the higher $q$ peak is observed from 80 to $120^{\circ} \mathrm{C}$.

On the basis of the fits to the Teubner-Strey scattering function, we conclude that the peak at lower $q$ derives from a bicontinuous microemulsion. In the range of $9-11 \%$ block 

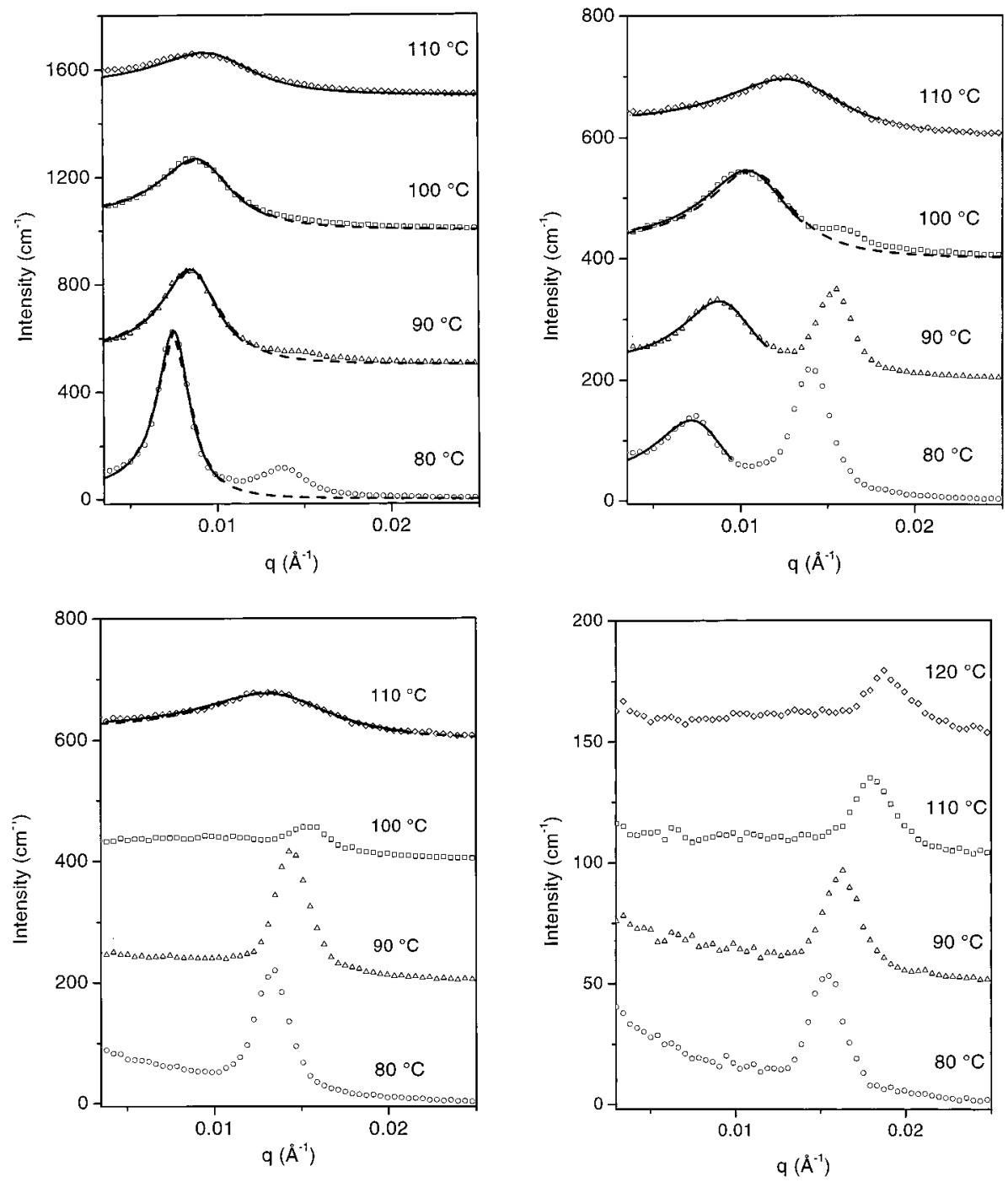

Figure 8. SANS patterns for the sample with (a) $9 \%$, (b) $10 \%$, (c) $11 \%$, (d) $12 \%$ copolymer, at the indicated temperatures. Fits to the TeubnerStrey structure factor are shown as solid lines. Dashed lines are fits over the full scattering range. Curves are offset for clarity.

copolymer, the intensity of the higher $q$ peak varies as a function of temperature and composition but the position remains constant at constant temperature. This suggests this second peak is not a higher order reflection but rather it is due to a second phase. The scattering data in Figures $8 \mathrm{a}-\mathrm{d}$ may be interpreted by assuming a region of phase coexistence between bicontinuous microemulsion and a lamellar phase. No higher order peaks were observed for the second phase, but the justification for assigning the higher $q$ peak to the lamellar phase will emerge during the subsequent discussion of the isothermal phase diagram.

The boundaries of the coexistence region appear to have both temperature and composition dependence. The channel of bicontinuous microemulsion covers a wider composition range at higher temperatures but appears to narrow as the temperature is lowered. At $110{ }^{\circ} \mathrm{C}$ scattering consistent with the existence of a bicontinuous microemulsion is observed between $9 \%$ and $11 \%$ block copolymer, but by $80{ }^{\circ} \mathrm{C}$ only scattering attributable to coexistence between bicontinuous microemulsion and lamellar phases was recorded. The region of pure microemulsion along the isopleth at $80{ }^{\circ} \mathrm{C}$ appears to be less than $1 \%$ wide in block copolymer composition.

At higher diblock concentrations, the isopleth displays lamellar ordering at low temperatures and disorder at high temperatures. A plot of SAXS data taken at $80{ }^{\circ} \mathrm{C}$ along the isopleth at PEO-PEP mass fractions from $21 \%$ to $100 \%$ is shown in Figure 9. The numbers associated with each scattering pattern refer to the mass fraction of diblock in the sample and the value of $q$ at the scattering maximum increases monotonically as the concentration of diblock increases. The $T_{\mathrm{ODT}}$ 's also increase monotonically from the lamellar-bicontinuous microemulsion coexistence region to the pure diblock ODT at 164 ${ }^{\circ} \mathrm{C}$. A plot of the principal $d$-spacing at $80^{\circ} \mathrm{C}$ as a function of diblock concentration is shown in the inset to Figure 9 including SAXS and SANS data. At $80{ }^{\circ} \mathrm{C}$, none of the phases display scattering due solely to the bicontinuous microemulsion. At $9 \%$ and $10 \%$ PEO-PEP, coexistence with a lamellar phase is observed and there are two symbols at that composition, one at $440 \AA$ and one at $840 \AA$. Interestingly, the sample with $11 \%$ diblock has a slightly larger principal $d$-spacing, $470 \AA$. The solid line is a power law fit to the lamellar data. The $d$-spacing varies with the volume fraction of diblock as $\phi_{\mathrm{PEO}-\mathrm{PEP}}{ }^{-0.9}$, close to the geometrical prediction of $\phi^{-1} .^{29}$

Isothermal Phase Diagram. The PEO/PEP*/PEO-PEP ternary phase diagram was characterized at $80{ }^{\circ} \mathrm{C}$ and is shown in Figure 10. For samples with greater than $20 \%$ block copolymer, SAXS measurements were used to identify the microstructure of the blend. This portion of the ternary phase diagram appears to be quite symmetric. The lamellar phase fills the middle of the phase diagram from pure block copolymer to nearly $20 \%$ diblock. When unequal amounts of homopolymer 


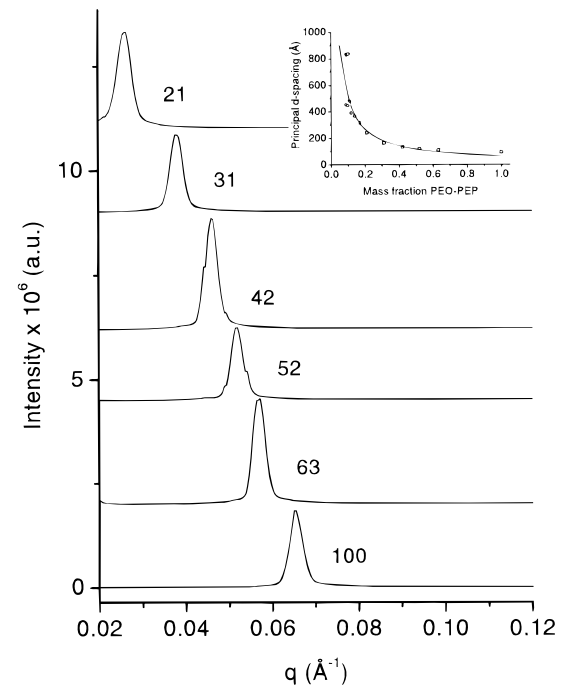

Figure 9. SAXS patterns for lamellar samples along the isopleth. Principal reflections are labeled by PEO-PEP mass fraction. Curves are offset for clarity. Inset: The $d$-spacing along the isopleth from SAXS (squares) and SANS (diamonds and circles) experiments. Coexistence between bicontinuous microemulsion (diamonds) and lamellae (circles) at $9 \%$ and $10 \%$ diblock is represented as two points at each composition. SAXS data points are shown as open squares. The solid line represents a power law fit, $d \sim \phi^{-0.9}$.

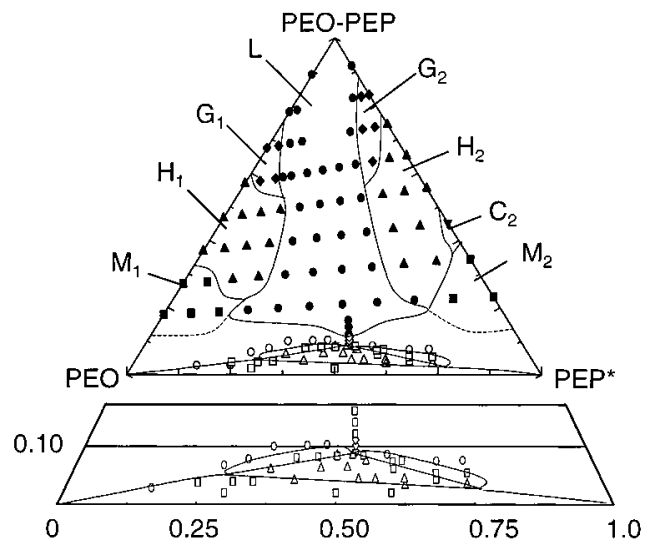

Figure 10. Isothermal phase diagram for the PEO/PEP*/PEO-PEP system at $80^{\circ} \mathrm{C}$. All symbols refer to actual data points. The subscript 1 indicates the majority phase in a particular structure is PEO, while 2 indicates it is PEP*. Dashed lines indicate the limits of data taken and not precise phase boundaries. Below $9 \%$ block copolymer, macrophase separation occurs and two- and three-phase equilibrium is observed. The portion of the diagram below $15 \%$ PEO-PEP is expanded below.

are added, the swelling of one of the blocks induces nonzero mean curvature at the interface between the PEO and PEP domains and the gyroid and hexagonal structures become stable. The $\mathrm{G}_{1}$ and $\mathrm{G}_{2}$ and $\mathrm{H}_{1}$ and $\mathrm{H}_{2}$ phases are nearly symmetrically disposed in the phase diagram with respect to the lamellar phase.

For mixtures containing less than $30 \%$ block copolymer, the only ordered phase observed is lamellar; it disorders directly to micellar solutions as the total volume fraction of either homopolymer is increased above 0.6 . The absence of any other ordered microstructure in this range of block copolymer concentrations is the reason for assigning the higher $q$ peak observed in the coexistence region identified in the isopleth phase diagram between $9 \%$ and $11 \%$ block copolymer (Figure 8) to the lamellar phase.

The phase behavior of samples with less than $10 \%$ block copolymer was characterized visually. The samples with $10 \%$ block copolymer were clear, viscous fluids. At lower diblock concentrations, samples that displayed multiphase equilibrium at $80{ }^{\circ} \mathrm{C}$ generally required at least 4 days of equilibration for three distinct phases to appear. The interface between the bicontinuous microemulsion and the PEO-rich phase is sharper than with the PEP*-rich phase. The middle, diblock-rich phase was usually slightly turbid which persists even after annealing for 2 weeks. Similar observations were made on samples thought to consist of bicontinuous microemulsion coexisting with a single homopolymer-rich fluid, suggesting this turbidity is intrinsic to samples with phase coexistence involving the bicontinuous microemulsion. It should be noted that the relative volume of one of the phases increases in samples that are richer in that component as would be expected in multiphase equilibrium.

\section{Discussion}

The experiments reported in the previous section were designed to aid in establishing a universal approach to describing the action of amphiphiles when mixed with otherwise thermodynamically incompatible compounds. The main advantages to working with the all-polymer system described here are the ability to enforce symmetric interactions between the two homopolymers, nearly equal interactions between the diblock copolymer and each homopolymer, and to access the disordered ternary mixture. These conditions are certainly not met with classical water-based systems. Judging from the symmetry of the PEO/PEP* phase diagram (Figure 3) and the similar appearances of the PEO/PEO-PEP and PEP*/PEO-PEP phase diagrams (Figures 6a and b), this ternary system satisfies these criteria. This also suggests it will be possible to make informative comparisons with the theoretical predictions for homopolymer/homopolymer/diblock copolymer phase diagrams. ${ }^{17,18}$

The isopleth phase diagram and the bicontinuous microemulsion phase have been investigated previously. ${ }^{14,15,22,35}$ Bates et al. ${ }^{14}$ used SANS, dynamical mechanical spectrometry, and static light-scattering experiments to characterize the isopleth for a higher molecular weight polyethylene (PE)/PEP/PE-PEP system. This blend undergoes a transition from a high temperature, disordered state to a macrophase-separated state at low block copolymer concentrations. At high block copolymer concentrations, the high temperature, disordered state undergoes microphase separation to a lamellar phase. The transition from bulk phase separation to microphase separation was studied as a function of block copolymer content and the identification of an apparent Lifshitz point was reported..$^{14}$ The Lifshitz point is the intersection in a ternary phase diagram where disordered, microphase-, and macrophase-separated phases coexist. Subsequent work on this system uncovered the existence of a bicontinuous microemulsion at the composition where critical scattering consistent with a Lifshitz point was observed. ${ }^{22}$ This called into question the existence of a Lifshitz point and spurred further work on characterizing the isopleth and bicontinuous microemulsions in homopolymer/homopolymer/diblock copolymer blends.

Hillmyer et al. ${ }^{15}$ performed a complementary investigation of three homopolymer/homopolymer/diblock copolymer systems and reported the phase behavior along the isopleth and SANS characterization of the bicontinuous microemulsion phase in each system. Many similarities were observed among these systems, and there was qualitative agreement with the predictions of mean-field theory, ${ }^{14}$ particularly in predicting the composition and temperature of the transition from microphase to macrophase separation in the higher molecular weight systems. However, mean-field theory does not anticipate the bicontinuous microemulsion. 


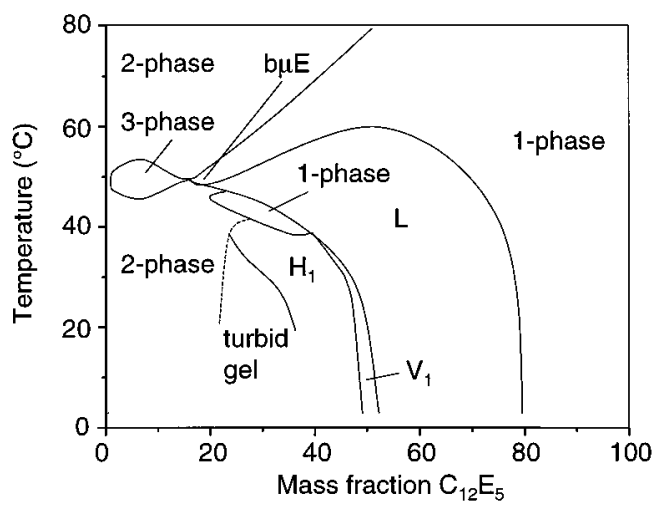

Figure 11. Isopleth for the water/oil/surfactant phase diagram (after Kahlweit and Strey ${ }^{30}$ ). At lower surfactant concentrations, multiphase equilibrium is observed. Below $40{ }^{\circ} \mathrm{C}$, a water-rich fluid containing dissolved surfactant is in equilibrium with an oil-rich fluid. Above 60 ${ }^{\circ} \mathrm{C}$, and the surfactant segregates preferentially to the oil-rich phase leaving almost pure water in the lower phase. At intermediate temperatures, the surfactant-rich bicontinuous microemulsion is a stable third phase. With higher surfactant concentrations, thermotropic and lyotropic liquid crystalline is observed. Above $80 \%$ surfactant a disordered fluid is present.

The general features observed in these studies have been identified in the isopleth shown in Figure 7. However, certain key differences should be noted. The first is the identification of three-phase equilibrium at low block copolymer concentrations and low temperatures. Previous work had labeled the macrophase-separated region generically as two-phase, largely because these studies were performed with high molecular weight polymers that effectively prevent equilibration due to their high viscosities and low diffusivities. Locating this threephase region is especially important for comparison to water/ oil/surfactant systems.

In their SANS investigation of the PEO/PE/PEO-PE system, Hillmyer et al. inferred the existence of a hexagonally ordered phase in a narrow region between the bicontinuous microemulsion and the lamellar phase, which was the stable ordered phase over much of the isopleth. The SANS experiments shown in Figure $8 \mathrm{a}-\mathrm{d}$ indicate a region of coexistence between lamellae and bicontinuous microemulsion. This raises the possibility that the hexagonal phase assignment was incorrect, and that actually phase coexistence between bicontinuous microemulsion and a lamellar phase was responsible for the occurrence of multiple SANS peaks.

The decrease in the amphiphilicity factor as the temperature decreases indicates that the bicontinuous microemulsion becomes more structured at lower temperatures. At $80{ }^{\circ} \mathrm{C}$ and $9 \%$ copolymer, an amphiphilicity factor of -0.93 was calculated for the bicontinuous microemulsion (in coexistence with the lamellar phase). This provides a clue as to why the channel of bicontinuous microemulsion narrows at lower temperatures. A lamellar phase has an amphiphilicity factor of -1.0 and as the amphiphilicity factor for the microemulsion approaches this value, the structure approaches that of the lamellar structure. Therefore, it appears that the lamellar phase becomes more stable at lower temperatures relative to the microemulsion. The obvious extrapolation is to the complete disappearance of the microemulsion channel at lower temperatures and the substitution of the lamellar phase at the tip of the three-phase window.

An isopleth for the water/ $\mathrm{C}_{14} \mathrm{H}_{30} / \mathrm{C}_{12} \mathrm{E}_{5}$ system is shown in Figure $11 .^{30}$ The amphiphile in this system, $\mathrm{C}_{12} \mathrm{E}_{5}$, is a nonionic surfactant where $\mathrm{C}$ represents a methylene group and $\mathrm{E}$ represents an ethylene oxide monomer. The polar and nonpolar groups in this surfactant have nearly equal volumes, similar to

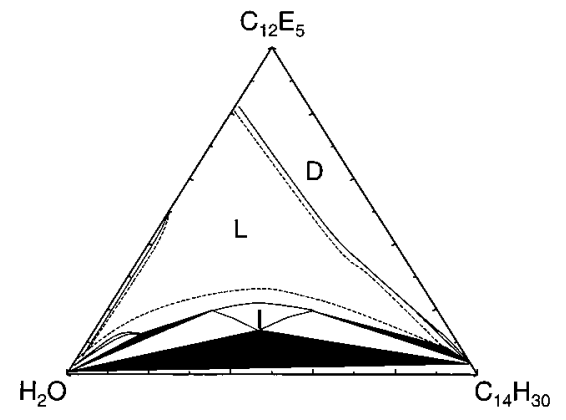

Figure 12. Isothermal phase diagram for the water/ $\mathrm{C}_{14} \mathrm{H}_{30} / \mathrm{C}_{12} \mathrm{E}_{5}$ system at $47.5^{\circ} \mathrm{C}$ (after Kunieda and Shinoda ${ }^{19}$ ). The central three-phase region (shown in black) represents equilibrium between water-rich and oilrich fluids and a surfactant-rich microemulsion (I). The three surrounding two-phase regions represent equilibrium between two of these phases. At higher surfactant concentrations, a lamellar (L) phase dominates most of the phase diagram except at low water and high oil concentrations, where an isotropic oil-rich fluid (D) is observed. Dashed lines represent phase coexistence boundaries.

the block copolymer used in this study. One striking difference between this phase diagram and the $\mathrm{PEO} / \mathrm{PEP}$ */PEO-PEP isopleth is the relatively narrow temperature range over which the three-phase window is observed. This highlights the strong temperature dependence of water as a solvent. Further evidence for this comes from the complex temperature and composition dependence of the ordered phase observed at higher surfactant concentrations. As the solvent properties of water change with temperature the curvature induced at the surfactant interface changes leading to thermotropic liquid crystal behavior, from $\mathrm{H}$ to a viscous, isotropic phase (V) to L. Another difference is $\mathrm{C}_{12} \mathrm{E}_{5}$ does not microphase separate until at least $20 \%$ water is added. Whereas adding homopolymers to a diblock copolymer lowers $T_{\mathrm{ODT}}$, adding water to a nonionic surfactant raises $T_{\mathrm{ODT}}$ due to the strong segregation between water and oil.

The PEO/PEP*/PEO-PEP system behaves in a qualitatively different manner. On the basis of the relative volumes of the two phases present at low PEO-PEP concentrations and 120 ${ }^{\circ} \mathrm{C}$, it appears that the block copolymer is slightly more soluble in PEO than PEP*. At $100^{\circ} \mathrm{C}$, the three-phase window is clearly visible and at $80^{\circ} \mathrm{C}$ it is even larger. Signs of the three-phase window are observed at even lower temperatures although diblock PEO crystallization restricts such experiments. This suggests that the homopolymers become comparable solvents for the diblock below $120{ }^{\circ} \mathrm{C}$, which leads to the stabilization of the third phase down to the lowest experimentally accessible temperatures. While there is evidence for some temperature dependence of the interactions in this system, the dependence is much weaker than for aqueous systems. The absence of temperature-driven structural phase transitions at higher block copolymer concentrations reinforces the assertion that the temperature dependence of the interactions between monomers is much weaker in the polymer system. To make comparisons with isothermal water/oil/surfactant phase diagrams, an experimentally determined, ternary phase diagram at $47.5^{\circ} \mathrm{C}$ based on the work of Kunieda and Shinoda ${ }^{19}$ is shown in Figure 12. Several differences between this ternary phase diagram and the polymeric one presented in this work are apparent. The first is the lack of variety in the ordered phases in the surfactant-rich region of the phase diagram at this temperature. The only ordered state observed has a lamellar structure and this is stable over a wide composition range.

The shape of the multiphase window in Figure 10 at PEOPEP concentrations less than $10 \%$ is consistent with those observed in aqueous surfactant systems. The three-phase triangle 
is almost symmetric with respect to the mass fractions of PEO and PEP*, suggesting that the strength of the interactions between diblock copolymer and each of the two homopolymers are similar at $80^{\circ} \mathrm{C}$. The bottom corners of the triangle extend close to the corners of the phase triangle, indicating the diblock copolymer is contained almost entirely within the middle phase. Finally, experiments at higher temperatures suggest the shape of the three-phase window is only weakly temperature-dependent, implying at most a slight temperature dependence in the interactions between EO and EP monomers. However, the shape of the multiphase window appears to reflect the symmetry of the $\mathrm{PEO} / \mathrm{PEP} *$ coexistence curve in the range of temperatures investigated even though the position of the plait point (a critical point in an isothermal two-phase window) is temperature dependent.

The faces of the three-phase triangle in Figure 10 (as in Figure 12) are bounded by regions of two-phase coexistence. Two of these regions have the bicontinuous microemulsion in equilibrium with a solution rich in PEO or PEP* and extend a few percent in diblock composition. The phase boundaries of both these regions continue into the three-phase window at the PEOPEP-rich corner of the triangle, consistent with Schreinemaker's rule, which requires that the boundaries of each homogeneous phase must intersect at the corner of the three-phase triangle in such a way that their extensions either both pass into the threephase triangle or both pass into the adjacent two-phase regions. ${ }^{31}$ This indicates the "groove" in the multiphase window caused by the existence of the three-phase region is relatively sharp, suggesting the domains are well segregated. ${ }^{32}$ This is consistent with the PEO/PEP* coexistence curve shown in Figure 3 which predicts very little mixing at $80{ }^{\circ} \mathrm{C}$. However, as noted in section 3 , the samples that are believed to be bicontinuous microemulsion in equilibrium with a homopolymer-rich fluid were slightly turbid. This may indicate that phase coexistence with the bicontinuous microemulsion requires long equilibration times. This may also explain why the lamellar $d$-spacing of the sample with 11\% PEO-PEP (shown in Figure 9) is larger than that with $10 \%$ block copolymer. At equilibrium the $11 \%$ sample may consist of bicontinuous microemulsion coexisting with lamellae but phase separation is kinetically hindered. These samples do not display any turbidity because the microemulsion and lamellar phases differ only in the concentration of PEO-PEP, which has nearly the same refractive index as the blend of homopolymers in both phases. Thus, even a coarsened blend of the two phases has a nearly homogeneous refractive index and does not scatter light. This suggests other two- and three-phase regions may be present at compositions with less than $11 \%$ diblock. However, due to the limitations inherent in visual measurements, three-phase equilibrium between, for example, lamellar, bicontinuous microemulsion, and oil-rich phases would appear as twophase equilibrium.

The existence of the gyroid phase in the PEO/PEP*/PEOPEP phase diagram is further evidence for the relatively weak interactions in this system. The gyroid phase is thought to exist only at modest segregation strengths; ${ }^{33}$ amphiphilic systems which are strongly segregated tend to undergo direct transitions from lamellar to cylindrical microstructures as the volume fraction of one of the components is increased. Alexandridis, et al. ${ }^{34}$ reported the observation of the gyroid phase in their investigation of the water- $p$-xylene- $\mathrm{EO}_{19} \mathrm{PO}_{44} \mathrm{EO}_{19}$ (where EO represents of monomer of ethylene oxide and $\mathrm{PO}$ represents a monomer of propylene oxide) phase diagram but the gyroid structure is stable over a relatively small area of the phase diagram. The $\mathrm{G}_{1}$ phase, where water forms the matrix, is observed only in a small region of the phase diagram whereas the inverted $\mathrm{G}_{2}$ phase, where xylene forms the matrix, occupies a larger region of the phase diagram. Furthermore, the phase diagram is highly asymmetric, which suggests water will exert a disproportionate influence on the phase diagram even when a larger amphiphile is used.

Our isothermal phase diagram is similar to one calculated by Janert and Schick ${ }^{17}$ (shown in Figure 2) and by Kielhorn and Muthukumar. ${ }^{18}$ With the exception of ordered cubic arrays of spheres, all the ordered microstructures associated with diblock copolymers were observed in our experimentally obtained phase diagram, including the gyroid phase that was not explicitly considered in their work. Our ternary isothermal phase diagram has a high degree of symmetry in the location of the ordered phases, as would be expected given the close similarities of the two homopolymer/diblock copolymer phase diagrams. The main differences between our experimental phase diagram and the theoretical phase diagram of Janert and Schick is that the lamellar phase occupies a much large region in the experimental phase diagram, and below $30 \%$ block copolymer, it is the only ordered microstructure. Whether this preference for the lamellar structure is due to the low molecular weight of the polymers has not been determined. Additionally, the ordered microstructures observed in the PEO/PEO-PEP and PEP*/ PEO-PEP phase diagrams persist to lower concentrations of block copolymer than is predicted by Janert and Schick. The value for $\alpha$, the ratio of the length of the homopolymer to the length of the diblock, is 0.17 in our system and the ordered states along the binary axes are predicted to terminate at approximately $80 \%$ block copolymer. This is likely due to the stronger segregation strengths in our system than that assumed by Janert and Schick in their calculation. On the basis of eq 1, $\chi N_{\text {PEO-PEP }}=35$ at $80^{\circ} \mathrm{C}$ compared to the value of 11 used in preparing Figure 2. Finally, very few examples of phase coexistence between microstructures were observed in the SAXS patterns. This suggests the regions of phase coexistence in this phase diagram are narrower than anticipated by theory.

\section{Conclusions}

The PEO/PEP*/PEO-PEP phase diagram presented here displays many of the characteristic features observed in water/ oil/surfactant phase diagrams. These include liquid crystalline phases at high diblock copolymer concentrations, coexistence between lamellar and bicontinuous microemulsion phases, and two- and three-phase equilibrium between isotropic fluids at low diblock copolymer concentrations. The differences in these two types of ternary phase diagrams are attributed to the strong effect water has in the water/oil/surfactant phase diagram. These differences include a much higher symmetry in the polymeric phase diagram, as evidenced by the symmetry of the isothermal ternary phase diagram, and much weaker temperature dependence in the interactions between the three components, as evidenced by the broad temperature window in which threephase equilibrium is observed. Polymer systems are thus valuable model systems for studying ternary phase diagrams with amphiphilic species.

Acknowledgment. We thank Travis Bailey, Dr. Robert B. Grubbs, and Todd Jones for assistance with the synthesis and purification of the materials used in this work. We also thank Dr. Martin Vigild for help with performing the SAXS and SANS measurements and Dr. Mary Beth Kossuth, Kasiraman Krishnan, Dr. Terry Morkved, and Prof. David Morse for useful discussions. This work was supported in part by the MRSEC Program 
of the National Science Foundation under Award DMR9809364, and the DOE through the Lockheed Martin Energy Research Corporation (Grant 19X-SX893V/DOE).

Supporting Information Available: Table of composition listed as mass fractions. This material is available free of charge via the Internet at http://pubs.acs.org.

\section{References and Notes} 525.

(1) Bates, F. S.; Fredrickson, G. H. Annu. Rev. Phys. Chem. 1990, 41,

(2) Hamley, I. W. The Physics of Block Copolymers; Oxford University Press: Oxford, 1998.

(3) Matsen, M. W. Phys. Rev. Lett. 1995, 74, 4225.

(4) Leibler, L. Macromolecules 1980, 13, 1602.

(5) de Gennes, P.-G. Scaling Concepts in Polymer Physics; Cornell University Press: New York, 1979.

(6) Han, C. C.; Bauer, B. J.; Clark, J. C.; Muroga, Y.; Matsushita, Y.;

Okada, M.; Tran-Cong, Q.; Chang, T.; Sanchez, I. Polymer 1988, 29, 2002.

(7) Fernandes, A. C.; Barlow, J. W.; Paul, D. R. J. Appl. Polym. Sci.

1986, 32, 5481.

(8) Winey, K. I.; Thomas, E. L.; Fetters, L. J. Macromolecules 1991, 24,6182 .

(9) Winey, K. I.; Thomas, E. L.; Fetters, L. J. Macromolecules 1992, 25,2645 .

(10) Koizumi, S.; Hasegawa, H.; Hashimoto, T. Macromolecules 1994, 27, 7893.

(11) Shull, K. R.; Mayes, A. M.; Russell, T. P. Macromolecules 1993, 26, 3929.

(12) Tanaka, H.; Hasegawa, H.; Hashimoto, T. Macromolecules 1991, 24,240 .
(13) Matsen, M. W. Macromolecules 1995, 28, 5765.

(14) Bates, F. S.; Maurer, W. W.; Lodge, T. P.; Schulz, M. F.; Matsen, M. W.; Almdal, K.; Mortensen, K. Phys. Rev. Lett. 1995, 75, 4429.

(15) Hillmyer, M. A.; Maurer, W. W.; Lodge, T. P.; Bates, F. S.; Almdal, K. J. Phys. Chem. 1999, 103, 4814.

(16) Broseta, D.; Fredrickson, G. H. J. Chem. Phys. 1990, 93, 2927.

(17) Janert, P. K.; Schick, M. Macromolecules 1997, 30, 137.

(18) Kielhorn, L.; Muthukumar, M. J. Chem. Phys. 1997, 107, 5588

(19) Kunieda, H.; Shinoda, K. J. Dispersion. Sci. Technol. 1982, 3, 233.

(20) Lang, J. C.; Morgan, R. D. J. Chem. Phys. 1980, 73, 5849.

(21) Schubert, K.-V.; Strey, R.; Kline, S. R.; Kaler, E. W. J. Chem. Phys. 1994, 101, 5343.

(22) Bates, F. S.; Maurer, W. W.; Lipic, P. M.; Hillmyer, M. A.; Almdal, K.; Mortensen, K.; Fredrickson, G. H.; Lodge, T. P. Phys. Rev. Lett. 1997, 79,849 .

(23) Hillmyer, M. A.; Bates, F. S. Macromolecules 1996, 29, 6994.

(24) Hajduk, D. A.; Kossuth, M. B.; Hillmyer, M. A.; Bates, F. S. J. Phys. Chem. 1998, 102, 4269.

(25) Wignall, G. D.; Bates, F. S. J. Appl. Crystallogr. 1987, 20, 28

(26) Flory, P. J. Principles of Polymer Chemistry; Cornell University Press: New York, 1953

(27) Teubner, M.; Strey, R. J. Chem. Phys. 1987, 87, 31951987.

(28) Selke, W. In Phase Transitions and Critical Phenomena; Domb, C., Lebowitz, J. L., Eds.; Academic Press: New York, 1992, Vol. 15.

(29) Skouri, M.; Marignan, J.; Appell, J.; Porte, G. J. Phys. II France 1991, $1,1121$.

(30) Kahlweit, M.; Strey, R. Angew. Chem., Int. Ed. Engl. 1985, 24, 654.

(31) Wheeler, J. C. J. Chem. Phys. 1974, 61, 4474.

(32) Kahlweit, M.; Strey, R.; Busse, G. J. Phys. Chem. 1990, 94, 3881

(33) Matsen, M. W.; Bates, F. S. Macromolecules 1996, 29, 1091.

(34) Alexandridis, P.; Olsson, U.; Lindman, B. Langmuir 1998, 14, 2627.

(35) Schwann, D.; Mortensen, K.; Frielinghaus, H.; Almdal, K. Phys. Rev. Lett. 1999, 82, 5056. 